\title{
My Diabetes My Way: clinical outcomes impact and user experiences for an electronic personal health record for diabetes
}

\author{
Authors: Scott Cunningham, Ana Pokrajac, Brian Allardice, Massimo Brillante, Lyn Wilson and Debbie Wake
}

\section{Aims}

My Diabetes My Way (MDMW) is an interactive website for people with diabetes and carers, with over 32,000 registrants in NHS Scotland. It contains multimedia resources for self-management and clinical records access (data from primary and secondary care, specialist screening services and laboratories). Features include: goal setting, communication tools and automated highly-tailored guidance based on results. We aimed to evaluate user experience, assess impact on outcome measures and model health economic benefits.

\section{Methods}

In 2015, an online evaluation survey was emailed to 3,979 active users of the MDMW electronic personal health record (ePHR) to assess their experiences and perceived benefits. In a cohort of long-term active users, clinical outcomes were assessed $\left(\mathrm{HbA}_{1 c}\right)$ and compared with age, gender, diabetes duration, treatment type and socioeconomic status-matched group.

\section{Results}

1,095 (27.5\%) active users completed the survey. Patients reported that MDMW improved their knowledge $(90.3 \%)$ and motivation $(89.3 \%)$. It allowed them to make better use of consultation time $(89.6 \%)$ and meant that they did not need to keep paper records $(84.4 \%)$ or phone their doctor for results $(85.2 \%)$. Users found graphs helpful to monitor changes (95.9\%) and help them meet their diabetes goals (83.5\%).

7,689 active users of MDMW had 3 or more years of follow-up data. There was a reduction in $\mathrm{HbA}_{1 c}$ in active users, with patients with type 2 diabetes not treated with insulin $(n=943)$ showing most significant changes (intervention cohort reduction from 59 to $54 \mathrm{mmol} / \mathrm{mol}$ (females) and 58 to $53 \mathrm{mmol} / \mathrm{mol}$ (males) within 1 year, versus matched control reducing from 58 to $57 \mathrm{mmol} / \mathrm{mol}$ (females) and 58 to $57 \mathrm{mmol} / \mathrm{mol}$ (males) during the same period). Intervention patients remained between $4 \mathrm{mmol} / \mathrm{mol}$ (females)

Authors: on behalf of My Diabetes My Way, Centre for Molecular Medicine, University of Dundee, UK and $3 \mathrm{mmol} / \mathrm{mol}$ (males) below their matched counterparts at 56 $\mathrm{mmol} / \mathrm{mol}$ after 3 years of follow-up $(p<0.001)$ sustained reduction. A return on investment of at least 6:1 has been modelled using UK Prospective Diabetes Study (UKPDS) predictions.

\section{Conclusion}

MDMW is a cost-efficient, population-based, self-management intervention with sustained impact on clinical measures. Current operating costs ( $₹ 2 /$ diabetes patient/annum) could offer significant cost savings through reduction of long-term complications and improved process measures. Patients report enhanced knowledge and understanding of diabetes and motivation to make positive changes. Improvements in $\mathrm{HbA}_{1 \mathrm{c}}$ are shown within 1 year of follow-up and are sustained at improved levels. The service is being implemented into other parts of the NHS.

\section{Conflict of interest statement}

Dr Wake, Mr Cunningham, Mr Brilliante and Dr Pokrajac are affiliated with the MyWayDigital Health Ltd (which is commercialising My Diabetes My Way outside Scotland) either as employees, shareholders or directors. 